\title{
Les styles de raisonnement médical à l'épreuve du concept de santé dans les années 1630-1670
}

Sarah Carvallo

\section{Résumé}

Le concept de santé constitue le noyau dur de toute théorie médicale, mais il s'enrichit d'une épaisseur propre à une époque, un lieu, une épistémologie et une métaphysique particulière. Dans la médecine moderne des années 16301670, d'un côté, il désigne un idéal caractérisé par la juste proportion entre les éléments, qu'étudie l'anatomie; de l'autre, une fréquence que repèrent de nouvelles technologies du savoir. Ces réaménagements scientifiques induisent une autre détermination de la santé dans le cadre d'une anatomie politique, qui vise non seulement à soigner, mais aussi à prévenir les épidémies et améliorer l'état général de la population. Cette alternative entre deux manières de généraliser l'individuel à travers deux objets anatomiques - l'humani corporis fabrica ou le body politick - permet de ressaisir l'épaisseur de la conception moderne de la santé opérant de façon plurielle dans la science et induisant un pluralisme feuilleté dans la médecine occidentale.

Mots clés: santé, règle, norme, anatomie, idéal, pluralisme, médecine moderne

\section{Summary}

The concept of health forms a main issue for the medical theory. In as much as it depends on a special time, place, epistemology and metaphysics, it gets a specific thickness. In the modern medicine of the years 1630-1670s, on the one hand, it delineates an ideal characterised by a just proportion, that anatomy studies; on the other hand, a frequency noticed by new technologies of

Sarah Carvallo, Université de Franche-Comté, Logiques de l'Agir (EA 2274), IHRIM ENS Lyon UMR 5317, Sarah.carvallo@ens-lyon.fr 
knowledge. Those scientific reorganizations bear a new conception of health in the frame of a political anatomy, which aims not only to cure, but also to prevent epidemics and optimize the general state of the population. The alternative to generalizing individual data through two anatomical objects - either through the humani corporis fabrica or the «politick body» - captures the thickness of modern health conception, which characterizes the cumulative ways of proceeding in science inducing a foliated pluralism in western medicine.

Keywords: health, rule, norm, anatomy, ideal, pluralism, modern medicine

Au milieu du dix-septième siècle, la médecine moderne juxtapose deux ontologies, deux épistémologies et deux axiologies de la santé, qui cherchent chacune à répondre à la tension entre le général et le singulier: comment passer des corps malades à la détermination de la santé? Il faut une règle, mais laquelle? Une première approche propose une règle (norma) géométrique sur le modèle de l'architecture pour estimer et redresser, une seconde une loi (rule, law) arithmétique à décliner en loi civile pour prévenir et améliorer. ${ }^{1}$ Cette tension met au jour l'épaisseur du concept moderne de santé, qui implique deux usages de la règle avec des enjeux théoriques et pratiques différents. Le concept de santé se décline alors en:

- un concept fin, non seulement au sens de Geertz en ce qu'il renvoie à une idée transhistorique et transculturelle de la santé, qui fait que la médecine à chaque époque et partout cherche à déterminer les critères de la santé et les moyens pour la conserver ou la restaurer. Mais aussi et surtout au sens de Ryle, en ce qu'il constitue le soubassement de tout acte ou geste anatomique, clinique ou thérapeutique, et s'avère donc constitutif du geste et du regard médical en tant que tel. ${ }^{2}$

- un concept épais, qui embarque une multitude de représentations, d'arrière-plans, de gestes, de manières de voir, connaître et soigner le corps, qui caractérise un style à une époque et en un lieu particuliers. En l'occurrence, la deuxième moitié du dix-septième siècle présente deux concepts alternatifs de santé, qui manifestent une ambivalence désormais constitutive de la médecine moderne occidentale.

En effet, le médecin ne se contente pas de soigner dans l'absolu. Il soigne tel ou tel malade, telle ou telle pathologie de telle ou telle façon, qu'il a au pré-

1 Sur les relations entre loi, norme, règle aux sens scientifique, moral et juridique, voir Daston et Stolleis 2008.

2 Sur les concepts épais et mince, voir Geertz 1973, et Ryle 1971. Ryle introduit cette distinction en prenant l'exemple du clin d'œil: est-il un tic ou un signe? Dans les deux cas, il suppose une action de la paupière, mais sa signification diffère. 
alable identifiée: il y a une logique épistémique, axiologique, institutionnelle et sociale du soin, qui embarque déjà une certaine posture médicale et une idée de la santé. Soigner, disséquer, prévenir implique toujours une qualité adverbiale, une précision sur la raison et manière (pourquoi et comment) dont on traite les corps. Or dans les années 1650-1670, la manière et la raison dont on soigne à Paris ne sont pas celles qu'on pratique et thématise à Londres ou en Irlande. Pourquoi apparaissent ces nouveaux objets, gestes et regards? Comment désempiler les strates sédimentées de la santé en ressaisissant les épistémologies de la règle ou de la norme mobilisées pour articuler des corps malades au concept de santé? À quel moment, où et pourquoi se constitue l'empilement, le feuilleté épistémique, qui caractérise le pluralisme scientifique à l'œuvre dans la médecine occidentale moderne? Il ne s'agit pas ici de suivre une perspective ethnographique au sens de Geertz, mais plutôt, selon la logique de Ryle, de faire émerger l'organisation logique d'un geste et d'un regard constitutifs de la médecine, lorsqu'elle détermine la santé à partir des corps malades pour les soigner. Il s'agit alors de ressaisir, dans la médecine moderne - et plus précisément en anatomie classique et politique -, la complexité de la description de la santé faisant système avec la philosophie, les mathématiques, l'architecture et la peinture, ainsi que les institutions et les technologies de son époque. Il faut alors repartir du problème que pose la détermination de la santé, pour analyser deux solutions alternatives, l'une classique sur le modèle de la géométrie et l'architecture déterminant la fabrique du corps humain, l'autre nouvelle sur le modèle de l'arithmétique et de la politique décrivant le corps politique.

\section{Le problème anatomique}

Toute science détermine un objet, qui intègre un ensemble de méthodes, de gestes ou pratiques, de représentations et d'objectifs pour répondre à un problème à la fois théorique et pratique. ${ }^{3}$ J'appelle problème anatomique la recherche d'une définition du corps à partir de sa structure (fabrica) pour constituer l'objet de la médecine dans sa double dimension: le corps sain et la classification des corps pathologiques que le médecin doit soigner. Certes,

3 «All sciences must deal with the problem of selecting and constituting 'working objects', as opposed to the too plentiful and too various natural objects. Working objects can be atlas images, type specimens, or laboratory processes - any manageable, communal representative of the sector of nature under investigation. No science can do without such standardized working objects $[\ldots], 19$. Atlases are systematic compilations of working objects. They are the dictionaries of the sciences of the eye. [...] atlas images make the science.» Daston et Galison 2007, 22. 
la médecine ne se réduit pas à l'anatomie, et le statut - interne ou externe à la médecine - de celle-ci suscite débat. Mais la Faculté de médecine de Paris entre 1630 et 1680 ou les débuts de l'arithmétique politique dans les années 1650-1670 avec William Petty considèrent l'anatomie comme la condition de sa scientificité ${ }^{4}$ À ce niveau de généralité, cet objet (humani corporis fabrica) intègre à la fois des faits (observés mesurés, expérimentés, etc.) et des valeurs - ici évoquées à travers le verbe déontique. Cet objet n'est pas donné, mais construit, défini, justifié par son insertion dans un contexte scientifique et culturel, une communauté, des technologies du savoir et des institutions, jusqu'à ce qu'il s'impose comme paradigme. Or dans les années 1640, les évidences anciennes se défont sous le coup de découvertes physiologiques, cliniques et anatomiques - en particulier, la physiologie harveyenne -, mais aussi par l'apparition de méthodes et objectifs médicaux nouveaux - notamment, avec l'iatromécanisme et l'iatrochimie - en parallèle avec les transformations des autres savoirs - physique, mathématique ou botanique - et des institutions - université, hôpital, État. Plus précisément, le problème anatomique se dédouble, pour ainsi dire, en un problème d'anatomie classique, posé depuis Galien et renouvelé par les perfectionnements de la dissection depuis Mondino dei' Liuzzi, et, un problème nouveau d'«anatomie politique», proposé par Petty pour améliorer la «fabrick» du corps politique. ${ }^{5}$

À ces deux niveaux, le «working object» de l'anatomie doit articuler la pluralité des corps sains ou malades avec le concept universel de santé. À quel corps sain la médecine se réfère-t-elle pour traiter les corps malades au double sens théorique et pratique du traitement? Comment découpe-telle ce corps-référence sur fond de l'ensemble des corps individuels? Comment le (re)présente-t-elle dans la dissection publique ou dans les planches anatomiques? Comment décide-t-elle de reconnaître dans le corps singulier la maladie ou la santé? Pourquoi certains médecins préconisent-ils d'appréhender le problème anatomique à l'échelle de la population? Pour valoir comme science, l'articulation entre le concept général de santé et la réalité plurielle des corps suppose une «règle»: dans l'anatomie classique, cette règle se détermine comme une «norma» qui permet de comparer les corps singuliers à la fabrique du corps humain. Dans l'anatomie politique, cette

4 Nous verrons comment, au contraire, Locke et Sydenham critiquent le risque d'une réduction de la médecine à l'anatomie et promeuvent une démarche clinique. Petty a étudié la médecine à Utrecht et Leyde dans un contexte cartésien qu'il critiquera par la suite; il connaît l'iatrochimie; il enseigne l'anatomie à Oxford en 1651; il intègre le cercle d'Hartlib dans les années 1647-1652, moment où paraissent ses premiers écrits originaux inspirés par le projet baconien d'une réforme des savoirs.

5 Petty écrit un traité sur «The Political anatomy of Ireland» en 1671. Lorsqu'il décrit le projet d'arithmétique politique au Comte d'Anglesey en décembre 1672, il utilise les expressions «political medicine» ou «political anatomy». 
règle se détermine par la mise en rapport arithmétique des catégories de corps selon une loi («rule») mathématique justifiant la mise en place de lois civiles. Ces deux règles caractérisent le feuilleté épistémique de la conception de la santé moderne, idéale ou populationnelle. Elles cristallisent deux styles de raisonnement scientifique distincts, qui s'intègrent dans une histoire longue non tant sous la forme d'une controverse ou d'un conflit, que d'un feuilleté structurant progressivement l'épaisseur de notre conception de la santé à travers deux objets anatomiques, l'«humani corporis fabrica» et le «body politick». Il faut repartir du problème classique posé dans les années 1630 pour comprendre l'émergence d'une alternative dans les années suivantes et du pluralisme scientifique, qui opère alors dans la définition de la santé. ${ }^{6}$

A partir du seizième siècle, l'anatomie classique se développe sous la figure de l'anthropologie, ${ }^{7}$ qui vise à produire la connaissance de la fabrique du corps humain par la dissection. Explorée par «les mains oculaires» de l'anatomiste, ${ }^{8}$ la figure corporelle révèle la nature et la perfection de l'homme: ${ }^{9}$ en tant que demeure de l'âme et image de Dieu, le corps exprime une perfection qui se traduit par sa beauté et la santé. Or c'est justement ce corps parfait qu'il s'agit de mettre au jour lors des dissections publiques, par opposition avec les dissections privées qui explorent la réalité des corps singuliers. ${ }^{10}$ Doyen de la Faculté de médecine à Paris, Jean Riolan (1577-1657) présente la dissection publique comme «la demonstration oculaire» ${ }^{11}$ de l'anatomie, et le manuel comme la démonstration rationnelle fondée sur l'autorité des Anciens. Tout le paradoxe de la dissection publique consiste à montrer un corps qui n'existe pas.

Le corps parfait de la science anatomique n'existe pas hic et nunc; il est encore moins donné dans les corps des condamnés à mort concrètement disséqués: il est choisi, construit à partir de la composition des parties de plusieurs corps.

Mais certes il faut avoir témérairement pris le premier corps qui s'est présenté pour la faire. Galien veut qu'on aille prudemment en l'eslection qu'on en faict [142]. [...] Que s'il faut faire choix de deux corps qui ne sont pas des plus propres, il sera tousiours plus à propos de re-

6 Seule l'alternative proposée par l'arithmétique politique est analysée ici. L'empirisme clinique britannique est évoqué. Dans d'autres textes, j'ai analysé le mécanisme et le vitalisme, et développé plus longuement l'empirisme anglais. C'est cette conjonction qui constitue le feuilleté épistémique caractéristique du pluralisme de la médecine moderne.

7 Carvallo 2017, Cunningham 2003, 208, French 1990, 1994, Mandressi 2003, 132-137, 2013, Pigeaud 1990, 399-400, 414, Sgarbi 2013, Siraisi 2001, 2012, Vons 2005, Wallace 1988, Wear 2000.

8 Riolan 1661, Advertissement au lecteur et auditeur, n.p.

9 Sur différentes conceptualisations de la perfection, soit absolue (Aristote), soit par degré (Arnaud de Villeneuve), soit par idéalisation (Galien), voir van der Lugt, 2011, en particulier 22-24.

10 Siraisi 2001; Jacquart 1998, 105; Grmek 1996, 8-9; Mandressi 2003.

11 Riolan 1661, 214. 
tenir celuy des deux: qui aura le moins d'age et de graisse, pour ce qu'il n'y a rien qui rende la dissection des nerfs si mal aysée que la graisse [143] [...] Mais pour faire une exacte démonstration, il faudroit avoir trois ou quatre corps, \& faire voir en l'un ce qu'on n'auroit sceu remarquer en l'autre. ${ }^{12}$

Il faut privilégier les corps minces et non mutilés. Après les avoir choisis, on les fait mourir à l'étouffée par noyade dans l'eau, plutôt que par la corde. Ensuite, un cadavre est consacré à la myologie, un autre à l'ostéologie «en un squelette bien monté et parfaictement bien adiusté», un autre à l'étude des viscères, un autre à celles des veines, un autre aux artères, et, enfin, un dernier aux nerfs, au cerveau et à la moelle épinière. L'ensemble de ces six cadavres forme une dissection publique en présentant la fabrique du corps humain, l'objet de l'anatomie. ${ }^{13}$ Pour sa part, Riolan, refuse de le représenter dans des planches anatomiques, car ce genre de figures «dépend d'une observation qui n'a jamais este faicte en disséquant [...] Au reste tous les Anatomistes ont tiré leurs peintures de Vesalius, \& il n'y en a presque point qui en ayent inventé des nouvelles». ${ }^{14}$ La détermination de cette fabrique sert alors de modèle pour repérer la maladie dans les corps singuliers:

«Il n'est pas difficile de connoistre les desordres, qui peuvent arriver en toutes ces parties, quand on a parfaitement connu de quelle sorte, elles doivent estre naturellement disposées.»15

ou encore, «la connoissance (des maladies) dépend de celle de la disposition naturelle des parties de tout le corps». ${ }^{16}$

Pour faire comprendre la détermination de cette fabrique, Riolan reprend un topos qui émaille les traités de médecine, mais trouve son origine dans la rhétorique cicéronienne. Comme Zeuxis peignait la femme parfaite à partir de la composition des beautés de plusieurs jeunes filles de Crotone, le médecin doit reconstituer dans sa dissection publique le corps humain sain à partir des cadavres disséqués. ${ }^{17} \mathrm{La}$ fabrique corporelle (dé)montrée résulte du

12 ibid., 142-144.

13 ibid., 144-145.

14 Il est vray que l'Anatomie représentée dans les pourtraicts donne du contentement à beaucoup de gens, voire se faict admirer à quelques-uns: mais certes, il arrive de ces images justement ce qu'Hippocrate disoit souvent: Beaucoup de gens admirent, mais peu sçavent. [...] Du Laurens a dict beaucoup de choses en faveur des peintures de l'Anatomie, mais on voit bien que cela n'a este que pour ce qu'il avoit rapporté celles de Vesalius dans son ouvrage, $\&$ les avoit faictes graver en taille douce toutes erronees, trompeuses et imparfaictes qu'elles estoient [...] (Riolan 1628-9, 125).

15 Riolan 1661, 274.

16 ibid., 291.

17 Zeuxis leur demanda aussitôt quelles belles jeunes filles se trouvaient à Crotone: on le conduisit d'abord au gymnase et on lui montra de nombreux jeunes gens de la plus pure beauté [...] Comme il admirait vivement en eux la grâce et les proportions: «Nous avons ici, lui dit-on, leurs sœurs encore vierges: tu peux, en voyant leurs frères, te faire une idée de leur beauté. - 'Présentez-moi donc, s'il vous plaît, dit Zeuxis, les plus belles de ces jeunes 
puzzle des parties de divers hommes, qui permet de passer des corps singuliers au corps humain universel.

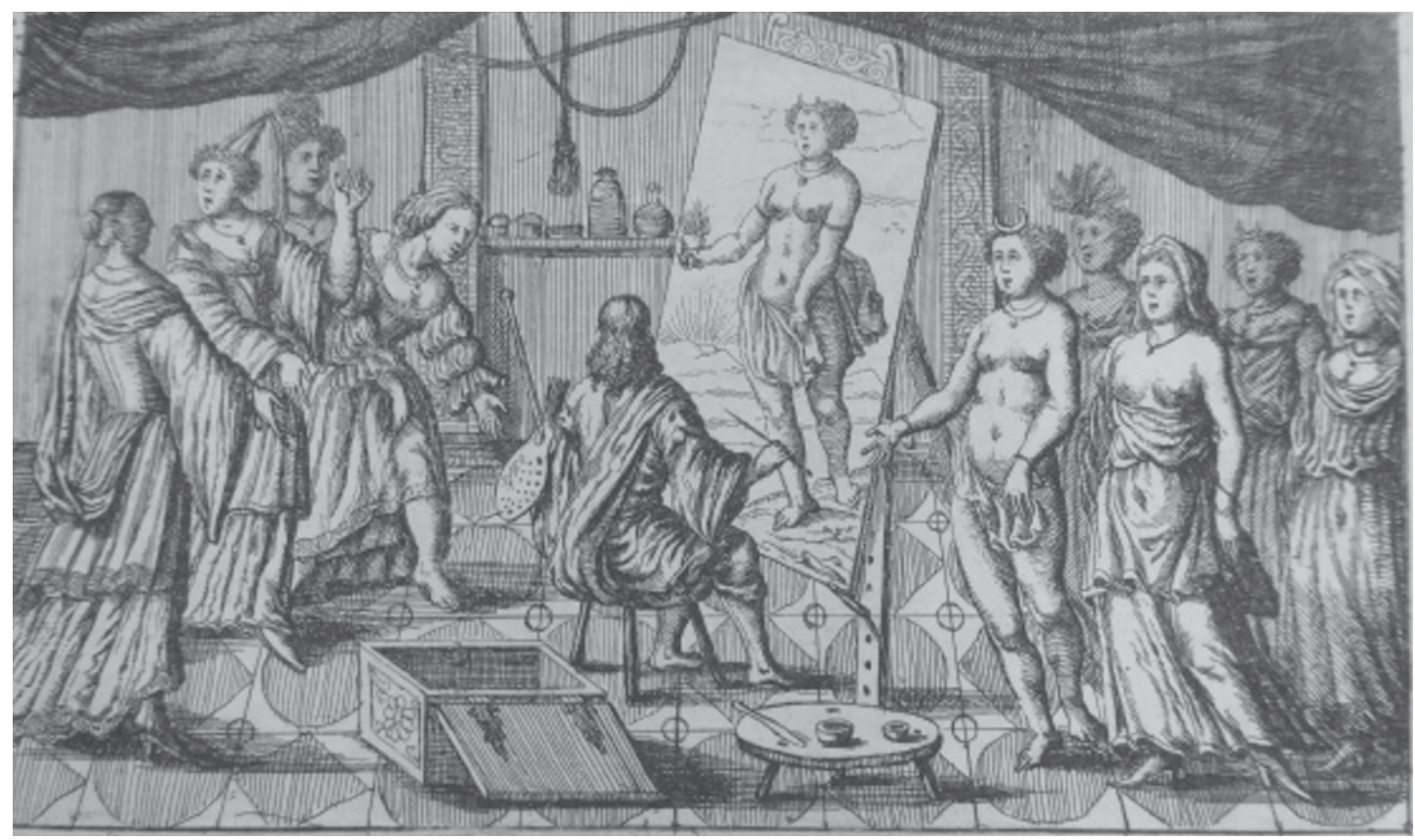

Zeuxis peignant Hélène, la plus belle des femmes, en combinant les parties des cinq plus belles femmes de Crotone. Source: Daniel LeClerc et Jacob Mangetus, Bibliotheca anatomica, Genève, 1685. Frontispice. Photographie de l'auteur, avec l'aimable autorisation de la Bibliothèque Municipale de Lyon, 22768.

Mais quel critère guide le choix et la composition? Comment concilier les règles de l'imitatio et de l'electio? Riolan ne trouve ce critère ni dans l'expérience empirique des corps, ni en physique ou mathématique, mais dans la littérature, la peinture et l'architecture. Homère, Vitruve, Varron ou Végétius, Sidoine Apollinaire décrivent la stature et l'apparence de ce corps. ${ }^{18} \mathrm{En}$ sculpture, Praxitèle, Polyclète et Debutades sicyonien lui servent de référence, lorsqu'il «[...] desire découvrir ce qu'il y a de plus beau, \& de caché dans la Medecine». ${ }^{19}$ En d'autres termes, le critère s'avère esthétique. L'objet scientifique converge avec la trajectoire de l'objet esthétique, en particulier

filles à titre de modèles pour le tableau promis: c'est ainsi que je pourrai faire passer dans une peinture inanimée la vivante vérité de la nature.»

Par décision officielle, ils réunirent les jeunes filles en un seul lieu, et autorisèrent le peintre à choisir librement parmi elles. Il n'en retint que cinq, dont maint poète nous a transmis les noms pour avoir obtenu les suffrages du maître le plus capable d'apprécier la beauté. Il ne crut pas pouvoir découvrir en un modèle unique tout son idéal de la beauté parfaite, parce qu'en aucun individu la nature n'a réalisé la perfection absolue. La nature, comme si elle craignait de ne pouvoir doter tous ses enfants en prodiguant tout au même, vend toujours ses faveurs au prix de quelque disgrâce.» (Cicéron, De inventione, II, 1-3).

18 Riolan 1661, 91-93.

19 Riolan 1661, l'Epistre du Manuel Anatomique, 4 et 6-8. 
du portrait en peinture ${ }^{20}$ et de l'édifice en architecture. L'alliance entre esthétique et médecine s'avère en effet constitutive de l'anatomie. ${ }^{21}$ L'autorisation pontificale de 1472 mobilise les deux justifications: la connaissance médicale et la beauté des portraits. Vésale insiste sur l'élégance des écorchés à reproduire sur les planches anatomiques; il choisit le meilleur lithographe, Jan Van Calcar, qui multiplie les références picturales pour persuader et convaincre de la vérité des images..$^{22}$ Le «souci de la beauté» traduit une stratégie argumentative. Même si Riolan récuse les planches anatomiques fallacieuses, il reprend le critère esthétique, dans la mesure où les jugements de vérité et de beauté sont analogiques.

Ce consensus s'enracine dans une allégeance aux sources antiques, qu'il faut «ressusciter» à la lumière des progrès modernes. ${ }^{23}$ Dans l'enseignement universitaire médiéval, l'épistémologie médicale se constitue historiquement à travers la convergence entre la médecine de Galien et la philosophie d'Aristote. ${ }^{24} \mathrm{~A}$ partir du treizième siècle, les universités de Bologne, Montpellier ou Paris établissent un cursus médical, qui amalgame ces deux traditions sous la figure du galénisme latin - non sans tensions, voire contradictions internes -, et détermine la médecine comme une partie de la philosophie naturelle selon deux axes: dans le prolongement de la Métaphysique d'Aristote 1070b28, elle étudie les relations entre santé et maladie afin de ressaisir la perfection du corps humain, ou dans la lignée des libri naturales, elle cherche à déterminer les conditions nécessaires pour maintenir l'équilibre entre les quatre éléments du chaud, sec, humide et froid. Même si la médecine de la première modernité présente de grandes divergences doctrinales, et si chaque université conserve une autonomie en matière de pratique et de théorie, le galénisme détermine un socle commun.

Aristote et Galien défendent tous les deux une analogie entre santé et beauté. ${ }^{25}$ Dans le De partibus animalium, Aristote considère le corps vivant

20 E. Pommier, Théories du portrait. De la Renaissance aux Lumières, Paris, Gallimard, 1999. Plus généralement sur l'alliance moderne entre esthétique et science, puis leur divorce, Daston et Galison,2007. En particulier, «Art and science converged in interwined judgements of truth and beauty.» 79. «Neither artists nor anatomists sensed any tension between the demands of truth and those of beauty; on the contrary, an ugly drawing was more than likely a false one. Like the discipline taught by the drawing schools, the halo of aesthetic appreciation surrounding the subject matter of botany and anatomy licensed naturalists and their illustrators to standardize and idealize objects drawn from nature.» ibid., 102.

21 Laneyrie-Dagen 2006, 196.

22 Vésale 2001, Préface; Temkin 1977, 44; Pigeaud 1985, 1995; Harcourt 1987.

23 Cunningham 2003, 115-116.

24 Carvallo 2017, Des Chene 1996, Garcia-Ballester 2002, 55-83, 75-102, Jacquart 1998, Nutton 2001, Pagel 1967, 332, Schmitt 1985, 1992, 23-27, 131, Sgarbi 2013, Siraisi 2001, 253.

25 Aristote, Topiques III 1, 116b 17-22; Galen, De placitis Hippocratis et Platonis, Kühn V, 448; De usu partium, Kühn I, 566; De Foetuum Formatione, IV, 695, Garcia-Ballester 2002, 129, 147, Pigeaud 1985. 
comme beau: il compare la nature à un grand architecte, qui voudrait construire une maison. Dans les Météorologiques, il introduit un autre argument au sujet de la perfection naturelle des êtres vivants sains: la vie ressemble à un cercle parfait, tout comme les éléments cosmologiques suivent des cycles. Ainsi l'eau tombe du ciel, s'infiltre dans la terre, percole dans les rivières et les mers, puis s'évapore. Dans les deux cas du vivant et du cosmos, le mouvement cyclique constitue la totalité à partir des parties et l'unité à travers le changement. Ce qui définit une perfection. ${ }^{26}$ Galien conçoit la santé comme «symmetria», la juste proportion entre les quatre éléments. Cette notion de proportion justifie le parallèle avec la beauté, car celle-ci se définit encore par le juste équilibre entre les parties, conformément au Canon de Polyclète, qui détermine chaque partie par un rapport proportionnel au tout. ${ }^{27}$ Dans le De anatomicis administrationibus, Galien explicite cette analogie entre santé et beauté à travers un parallèle entre médecine et architecture. Certes, la complexion parfaite (eukrasia) n'existe pas plus que la santé idéale. Toutes deux caractérisent une perfection ou un idéal. ${ }^{28} \mathrm{Ce}$ qui ne remet pas en cause néanmoins la réalité de la santé dans les corps individuels: selon l'échelle qui va de la santé parfaite à la maladie, les corps sains divergent seulement légèrement (parum lapsi) de la perfection à cause de lésions insensibles et se distinguent des corps neutres (neutra) qui sont potentiellement malades..$^{29}$ Un concept trop absolu de santé supposerait que nous sommes tous malades, ce qui serait absurde.

Comment thématiser alors la règle qui permet de passer de la pluralité des corps sains, malades, imparfaits, à l'universalité de la fabrique du corps humain, parfait, beau et sain?

\section{La règle de la santé idéale ou parfaite}

En s'inscrivant dans une stricte allégeance à Galien, Riolan cherche à déterminer la forme parfaite du corps humain en reprenant l'analogie galénique entre santé et beauté, mais en la conciliant avec les Ecritures. ${ }^{30}$ Riolan intro-

26 C'est encore la définition de l'Encyclopédie, qui traite de la perfection en métaphysique. $\S$ Perfection, [Métaphysique], Encyclopédie, ARTFL Encyclopédie Project, t. 12, 352.

27 Galen, De placitis Hippocratis et Platonis, Kuhn V 3, 16. Le terme canon se retrouve en arabe aussi bien dans un contexte politique (avec Soleiman dit Al Qanuni) que médical (par exemple, Le Canon d'Avicenne ou Al-Qanun fil-tibb, Le canon de la médecine, d'AlBiruni), J. Ruby 1986, notes 19 et 21.

28 Van der Lugt 2011, 17-18.

29 Galien, Exhortation à l'étude de la médecine. Art médical, IV 6-7. Voir les commentaires de van der Lugt 2011, en particulier 31.

30 Riolan 1649, 3-4, 16-18. 
duit alors un topos issu de la tradition architecturale antique en paraphrasant la description vitruvienne de l'homme parfait dans le De Architectura III 3:

il faut aussi en [du corps] reconnaître la forme, laquelle à cause de la justesse et symétrie des parties doit servir de règle et de mesure au tout, d'où vient qu'Hippocrate au I. Livre des Articles, a dit, que l'homme a obtenu un corps très bien compassé, et il est très vrai que la figure ronde la plus excellente de toutes les autres, est très manifestement remarquée au corps de l'homme lorsqu'il est couché sur son dos et a ses bras et jambes ouvertes: car si on vient à mettre l'une des branches d'un compas droit au nombril, et que de l'autre on tournoie alentour du corps, on touchera les extrémités des doigts, tant des pieds que des mains. La figure carrée se trouve aussi au même corps si la longueur, qui est depuis le sommet de la tête jusques à la plante des pieds est appliquée à celle qui est du bout d'une main à l'autre lorsque les bras sont ouverts, de sorte que les anciens semblent avoir ordonné avec raison qu'on gardât en tant qu'il se pourrait en tous chefs d'œuvre la proportion de nos corps et de chacune de leurs parties, \& bien que ce fût une règle générale pour toute sorte de bâtiments; si est ce qu'elle était sur tout fort exactement gardée en la structure des temples des Dieux [...]. (Riolan 1628-9, 8. Je souligne)

Dans le De Architectura, Vitruve établit une théorie du corps humain parfait à travers le calcul des proportions. ${ }^{31} \mathrm{Il}$ pose une équivalence entre le corps et l'édifice, l'anatomiste et l'architecte grâce au concept de symmetria. Riolan assume ce parallélisme: l'inscription du corps dans le cercle ou le carré, figures géométriques parfaites, garantit la beauté de la forme corporelle. Cette idée géométrique du corps (proportion des parties, inscription dans le cercle et le carré) vaut comme règle - en français, norma en latin - pour juger les corps de chair qu'ausculte le médecin en les rapportant à cette référence idéale, tout comme elle sert de règle pour déterminer la construction des édifices.

L'on doit donc considerer en l'homme, le sexe, la substance du corps, le temperament, la grandeur, la couleur, la forme, ou la figure, et voir de quelle sorte toutes ces choses sont en un homme parfait, afin que cela nous serve d'une regle asseurée pour connoistre ce qui manque à ceux qui s'éloignent de la perfection. (Riolan 1661, 86. Je souligne).

Riolan convoque la règle au sens de l'outil qu'utilise l'architecte pour tracer le dessin des édifices. A l'âge classique, les termes associés à la norme n'apparaissent guère dans les textes médicaux en langue française. Ni le Dictionnaire de Furetière (1690) ni le Dictionnaire de Trévoux (1721) ne les utilisent comme entrée; l'Encyclopédie n'a pas d'entrée «Norme» et pour «normal» ne mentionne pas de sens médical, mais seulement géométrique:

Normal adj. adj. (Géom.) une ligne normale, en Géometrie, est ce que l'on appelle autrement \& plus ordinairement une perpendiculaire. Voyez Perpendiculaire. Encyclopédie, ARTFL Encyclopédie Project, t. 11,228.

31 Vitruvius 2015, LV-LXV, 160-169, Gros 2006, 83-84, 273, Caye 2001, Raven 1951, Tobin, $1975,307,321$. 
Cette lacune est significative: la généralisation du terme et du concept de norme dépend en réalité du développement de la pensée statistique..$^{32}$ Dans la langue française, le mot «norme» et la science des statistiques vont de pair et caractérisent un certain régime de rationalité absent au début du dix-septième siècle. Les médecins ne connaissent pas alors de décalage entre normativité et normalité, valeur et fait: la santé se trouve tout entière du côté axiologique, comme degré de perfection des corps vivants, mais aussi tout entière du côté ontologique comme réalité de référence à laquelle rapporter les corps singuliers. Dans la perspective d'une ontologie aristotélicienne structurée par l'échelle des êtres, les vivants s'ordonnent selon leur degré de perfection, qui correspond à leur degré d'être. Cette échelle ne se détermine pas de façon empirique, mais métaphysique fondée sur l'analogie entre le Fabriquant et les créatures, et sur les analogies structurelles entre les êtres. Comme le souligne Riolan dans le cas de la médecine, l'idée de l'homme parfait vaut comme référence ou modèle pour comparer les corps singuliers en chair et en os, afin de décider de leur bonne ou mauvaise santé.

Cette référence se nomme «règle» ou «canon» et s'inscrit dans un registre esthétique, architectural et médical. Elle exprime le sens du terme latin «norma», qu'utilise Vitruve mais qui ne fait pas français. ${ }^{33}$ Le glossaire d'architecture le traduit par «équerre»: "C'est ce que Vitruve appelle norma», ${ }^{34}$ ou encore, par «règle» pour désigner l'instrument de mesure utilisé par les artisans..$^{35}$ Perrault le transpose par «règle», lorsqu'il traduit le De Architectura sur ordre de Colbert pour établir un «Vitruve François». ${ }^{36}$ Dans l'Encyclopédie, le médecin Jaucourt conserve la terminologie française en esthétique:

Règle la, (Sculp. antiq.) C'est ainsi qu'on nomme une fameuse statue antique de Policlete, l'un des plus grands sculpteurs de la Grèce. Les regles de l'art étoient si bien observées dans cette statue, qu'on l'appella par excellence la Regle. (Jaucourt, Encyclopédie, ARTFL Encyclopédie Project, t. 14, 25)

Vitruve considère la «norma» comme nécessaire à l'architecte, qui doit être instruit en arithmétique et géométrie (Vitruve I, 1,4) pour déterminer les opérations modulaires permettant d'établir les dimensions et proportions des édifices et des colonnes (III, 3, 12; III, 3, 7, III, 8-12). Déjà le terme «norma» opérait un passage du grec au latin, puisqu'il traduit le mot grec «gnômôn» à travers un synonyme étrusque, qui désigne ce qui sert de régulateur ou de

32 Rey 2006, t. 2, 2393.

33 Sur l'importance de faire français et de constituer le français comme langue de science et de goût, style français, institution royale capable de dépasser les frontières du Royaume, voir Fumaroli, 1984.

34 D'Aviler 1691, 573.

35 D'Aviler 1691, 799.

36 Perrault 1674, Avertissement, n.p. et Perrault 1673, 2, n. 3. 
règle. ${ }^{37}$ Rappelant cette source, Budé souligne le débat à l'œuvre dans la traduction française de la norma vitruvienne entre équerre et règle:

Les Latins, dit Budé, usent de cette diction norma, comme les grecs de gnomon, pour exprimer l'instrument par lequel les artisans dressent les angles de leurs besongnes: nous l'appellons équerre. [...] La partie d'architecture qui enseigne cela est ditte gnomonique, que Jan Martin ne devoit pas tourner régularité [...] (Bertin et Gardet 1556, 19).

Le gnômôn caractérise l'outil de la géométrie chez Euclide. Il requiert le $k a$ nôn, une tige, un roseau, ou, plus généralement, «toute barre longue et droite», qui sert à l'opération de tracer droit et vérifier l'alignement au cordeau; avec Polyclète, il passe en sculpture pour désigner la juste proportion. Vitruve l'utilise pour décrire les règles, que doivent utiliser les techniciens, au double sens de l'instrument et de l'obligation. Dans le célèbre passage où il assigne les proportions parfaites du corps humain («l'homme de Vitruve», que cite Riolan, cf. supra) comme référence pour dessiner les édifices, il écrit:

Le centre du corps humain est en outre par nature le nombril; de fait, si l'on couche un homme sur le dos, mains et jambes écartées, et qu'on pointe un compas sur son nombril, ou touchera tangentiellement, en décrivant un cercle l'extrémité des doigts de ses deux mains et de ses orteils. Mais ce n'est pas tout: de même que la figure de la circonférence se réalise dans le corps, de même on y découvrira le schéma du carré. Si en effet mesure est prise d'un homme depuis la plante des pieds jusqu'au sommet de la tête et qu'on reporte cette mesure sur la ligne définie par ses mains tendues, la largeur se trouvera être égale à la hauteur, comme sur les aires carrées à l'équerre (quemadmodum aerae, quae ad normam sunt quadratae). (Vitruvius, De Architectura, III, 1, 3, 1990, 7. Je souligne)

P. Gros souligne le sens géométrique de cette norma-équerre, qui précise la quadrata designatio de l'homo quadratus ou bene figuratus. Elle est à la fois naturelle, parfaite et géométrique, bref idéale. Plus loin, Vitruve l'applique néanmoins à résoudre des problèmes concrets, comme l'inclinaison des entablements des façades (III 5,13), qui vise à corriger les effets de perspective «comme il a été prescrit ci-dessus, elles apparaîtront alors à nos yeux d'aplomb et à l'équerre (ad perpendiculum et normam)», ${ }^{38}$ ou le dessin des cannelures des colonnes (III 5, 14),

Columnarum striae faciendae sunt quatuor et viginti, ita excavatae, uti norma in cavo striae quum fuerit coniecta, circumacta anconibus, striarum dextra ac sinistra angulos tangat, cacumenque normae circum rotundationem tangendo pervagari possit. (Vitruvius, De architectura III, 5, 14 2015, 200)

Les colonnes doivent compter 24 cannelures, refouillées de telle façon que si l'on place une équerre dans le creux de l'une d'elles, et qu'on la fasse pivoter, elle touche de ses deux branches les arêtes droite et gauche des listels intermédiaires [...] (trad. Pierre Gros, Je souligne)

37 Cassin 2004, § Gnômôn, Metron, kanôn), 712.

38 Gros 32. 
L’ambiguïté entre une norme idéale et une réalité décalée se retrouve dans le paradoxe de la «basilique normale» entre un idéal théorique, qui n'a jamais existé mais qui sert de référence aux édifices qu'il faut construire, et la genèse d'une structure, qui doit s'accommoder des espaces et bâtiments existants de façon pragmatique. Forme canonique, schéma normatif ou archétypal d'un côté, et pratique expérimentale de l'architecture dans la négociation des contraintes, de l'autre, ${ }^{39}$ toutes deux rapportées à la figure du corps humain déterminée par des proportions parfaites géométriquement. À la Renaissance, reprenant la statue de Polyclète et rédigeant en latin, Vésale applique le canon ou la règle à l'anatomie en explicitant sa fonction de modèle régulateur lors des dissections publiques, qui doivent exhiber la vérité du corps humain sain:

C'est pourquoi il convient de présenter à la dissection publique, pour chaque sexe, un corps bien tempéré, d'âge moyen, comme on peut rapporter à la statue de Polyclète tous les autres corps. ${ }^{40}$

Même s'il critique Vésale en général, Riolan reprend ce schème classique. ${ }^{41}$ Son successeur à la chaire de physiologie de la Faculté de médecine de Paris, Claude Perrault (1613-1688) prolonge encore ce topos tout en y intégrant le paradigme de la circulation sanguine que Riolan récusait, mais désormais admis: ${ }^{42}$ son œuvre incarne l'alliance entre architecture et médecine, dans le cadre des nouvelles institutions étatiques et scientifiques - les Académies lancées par Colbert. ${ }^{43} \mathrm{Il}$ fait partie du petit conseil de Colbert aux côtés de Le Brun, premier peintre du Roi, et Le Vau, avec le soutien de son frère Charles, premier commis de la Surintendance des Bâtiments du Roi de 1664 à 1680, il promeut les «véritables règles du beau et du parfait dans les édifices» ${ }^{44}$ pour déployer «une architecture d'Etat à valeur universelle», ${ }^{45}$ qu'il applique notamment dans la façade du Louvre. Il s'engage aussi dans les plans de l'Observatoire, dont il dirige la construction en $1667 .{ }^{46}$ Au niveau esthétique, l'homme de goût reconnaît ces règles établies scientifiquement à travers le plaisir:

[...] la Beauté n'ayant guere d'autre fondement que la fantaisie qui fait que les choses plaisent selon qu'elles sont conformes à l'idée que chacun a de la perfection, on a besoin de regles qui forment et qui perfectionnent cette idée. (Perrault 1673, Préface n.p. Je souligne.)

39 Gros 2006.

40 «Corpus itaque publicae sectioni adhiberi convenit in suo sexu, quam temperatissimum et aetatis mediae, ut ad hoc tanquam ad Policleti statua alia corpora possis conferre.» Vésale, 1543-2001, Livre V, 19. Harcourt 1987; Siraisi 2001, 302.

41 Mani 1974.

42 Picon 1989, Mazauric 2007, 35-58, Des Chene 2005. Perrault devient Docteur Régent en 1652, professeur de physiologie, puis professeur de pathologie en 1653.

43 Salomon-Bayet 2008, 30-32, 71-73; Marauric 2007, 35-58, 177-208.

44 Perrault 1673, Préface n.p.; Hermann 1980, 113, 121-126.

45 Lemerle 2009.

46 Petzet 1967.

Gesnerus 76 (2019) 
Et ces règles architecturales proviennent du corps humain, structure parfaite, «La plus fine et la plus délicate de toutes Architectures». ${ }^{47}$ L'homme est une

Mesure et [d']un Module, de même qu'on en fait en Architecture: et considerant tout l'Univers comme un grand et superbe Edifice, qui a plusieurs appartemens d'une structure differente, on a choisi les proportions du plus noble pour regler tous les autres. (Perrault, in Histoire de l'Académie, 1773, t. 3, Préface xviii. Je souligne.)

Ainsi le concept de règle articule métaphysique, morale, médecine et esthétique, vérité, perfection, santé et beauté. Et cette alliance s'ancre dans l'héritage latin, plus que grec, de la médecine et de l'architecture françaises classiques, qui caractérisent l'homme de goût:

[...] et quand Ciceron donne des exemples d'une science qui a une vaste etenduë, il allegue l'Architecture, la Médecine et la Morale. Platon a esté dans le mesme sentiment quand il a dit que la Grece toute sçavante qu'elle estoit de son temps, auroit eu de la peine à fournir un Architecte. (Perrault 1673, 2)

Quatre siècles plus tard, Canguilhem souligne encore l'ambivalence intrinsèque, qui creuse le rapport entre norme et santé:

Quand on sait que norma est le mot latin que traduit équerre et que normalis signifie perpendiculaire, on sait à peu près tout ce qu'il faut savoir sur le domaine d'origine du sens des termes norme et normal, importés dans une grande variété d'autres domaines. Une norme, une règle, c'est ce qui sert à faire droit, à dresser, à redresser. (Canguilhem 1966, 177).

\section{La santé comme fait, la règle comme rapport arithmétique}

Néanmoins, à la même période, des médecins modernes critiquent cette référence à un idéal pour déterminer la santé et préconisent une autre approche fondée sur les faits purs, soi-disant débarrassés de métaphysique. ${ }^{48}$ Cette contestation s'opère essentiellement en Angleterre et en Irlande, soulignant la pluralité des rationalités médicales opérant à travers des règles différentes. D'une part, des médecins empiristes comme Sydenham (1624-1689) ou Locke (1632-1704) revendiquent l'observation clinique des cas pathologiques pour élaborer une classification des symptômes sur le modèle des typologies botaniques. ${ }^{49}$ Ils s'opposent explicitement au galénisme ${ }^{50}$ et promeuvent davantage le modèle hippocratique du traité des Airs, des Eaux et des Lieux. Souvent présenté comme l'Hippocrate anglais, Sydenham considère qu'un

47 Histoire de l'Académie, 1733, t. 1, 143.

48 Banta 1987, Carey 1997, Cook 1986, 1990, 1994, 2008, 2010, Dear 2008, Pomata 2010, Pomata et Siraisi 2005, Sgarbi 2013; Wear 2000.

49 Anstey 2005, 2011, Carvallo, 2016, Crignon 2013, Hess, Mendelsohn 2010, Romanell 1984, Waldow 2010, Walmsley 2000.

50 Cook 2010, Dear 2005, Wear 2000, 36 
bon médecin n'est pas un philosophe, qui connaîtrait la santé en tant qu'idée ou idéal du corps sain; ${ }^{51}$ il ne peut pas non plus accéder aux causes pathogènes; ${ }^{52}$ il s’intéresse à la «constitution» des paramètres environnementaux, des faits épidémiologiques, des conditions de vie, des saisons et du climat; ${ }^{53}$ il exerce une action préventive et thérapeutique, qui s'évalue de façon pragmatique en fonction de ses succès. Comme Locke, Sydenham relativise l'usage de l'anatomie ${ }^{54}$ et récuse la place des concepts théoriques en médecine au profit d'une attitude empirique et pragmatique. ${ }^{55}$ Chaque patient présente une histoire singulière hic et nunc, mais l'agrégation de plusieurs traits communs chez différents sujets permet de constituer des catégories de cas. Un cas constitue un paradigme, qui vaut comme référence pour classifier et interpréter les symptômes des divers patients. En déterminant un ordre des maladies, une historia morbi, des conditions nécessaires, ces classifications produisent les conditions théoriques d'une nosologie, que François Boissier de Sauvages thématisera - non sans reconnaître sa dette envers Sydenham. ${ }^{56}$

En lien avec cette épistémologie, les médecins anglais critiquent le monopole universitaire et considèrent que la médecine prend place au chevet du malade, soit en ville, soit à l'hôpital, qui doit collecter les informations et devenir aussi un lieu de formation Nosocomium Academicum. ${ }^{57}$ Ils promeuvent ainsi «un ensemble d'activités et de pratiques, plutôt que des concepts purs $»^{58}$ et participent à la reconfiguration de l'organisation hospitalière. ${ }^{59}$ En passant d'un programme anatomique fondé sur l'idéal du corps humain parfait à un projet clinique et classificatoire sur le modèle de la botanique, les médecins anglais transforment les exigences scientifiques: ils ne visent plus à expliquer les causes, mais à décrire les symptômes au niveau des individus et des populations pour améliorer la nosologie. Ils s'intéressent aux histoires des patients, à la compilation de cas sans hypothèses préliminaires, à l'évaluation des traitements et de leurs effets.

D’autre part, des mathématiciens, des médecins et des philosophes développent une nouvelle méthode sous le nom d'arithmétique politique, selon l'expression proposée par William Petty autour de $1672 .{ }^{60}$ Cette approche ne

51 Anstey 2011, Cantor 2002, Cunningham 2002.

52 Sydenham 1848-50, 72; Locke 1979b, 585-586, Anstey 2011a, 46-69.

53 Sydenham, 1848-50, I 72, II 9, Cunningham 1989, Duchesneau 1998, 241-250, Meynell 2006.

54 Carvallo 2017, Dewhurst 1958, Wilson 1988; Wolfe 1961.

55 Anstey 2011, Rütten 2002, Wear 2000.

56 Hess, Mendelsohn 2013.

57 Mc Cormick 2009, 68.

58 Cook 2010, 9-10. Ma traduction.

59 Lawrence 1996, 238, Martensen 2002.

60 Slack 2004. 
concerne plus les corps individuels qu'auscultent les empiristes, ni un corps idéal que déterminent les professeurs d'anatomie, mais des catégories de corps rapprochés selon des critères tels que leurs pathologies, les causes de décès, les lieux d'habitation, les professions, l'âge, le sexe. À la différence du paradigme topique de la botanique, ces catégories constituent des séries compilées sur une année, comparables avec celles des autres années ou d'autres lieux. Elles abandonnent la logique narrative de l'historia morbi pour celle du tableau. Ces nouvelles notations modifient aussi le concept de santé: il ne désigne plus l'état objectif d'un individu dans une constitution déterminée ou une perfection ontologique et axiologique, mais se conçoit à partir d'un état fréquent dans une population en un lieu et un moment donnés. ${ }^{61}$ Elles veulent faire émerger une santé publique sans théorie, obtenue par l'extraction des informations données par les séries grâce au repérage de rapports arithmétiques stables. L'accumulation des données permet ainsi d'extraire l'information utile - utile, non pas immédiatement, mais a posteriori parce qu'elle sera agrégée et comparée - et négliger le reste. Le travail des «searchers» - en général, des femmes soignantes - qui remplissent les registres paroissiaux consiste à établir le matériau des séries - avec des erreurs, qu'il faut ensuite détecter et corriger sur le papier. Le travail des médecins, mathématiciens ou politiques consiste à repérer des relations arithmétiques significatives entre les éléments des séries, à corriger les erreurs de collecte. Il y a un cercle épistémique, puisqu'il faut attendre la constitution des autres séries pour constater a posteriori et par un autre sujet - médecin, mathématicien, ou politique - que celui qui a effectué la compilation, la présence de relations constantes, sur lesquelles l'hôpital ou l'État pourront agir.

D'une façon générale, Jack Goody a montré comment la raison graphique constitue une forme de rationalité particulièrement efficace. ${ }^{62}$ Plus précisément, Hess et Mendelsohn ont souligné l'importance des «technologies de papier» pour organiser les cas pathologiques en séries et produire du savoir; ${ }^{63}$ tout à l'heure, les dissections publiques et les planches anatomiques permettaient d'imposer certaines représentations du corps sain et beau; à présent, les tables et registres permettent d'établir des rapports arithmétiques entre différentes catégories de phénomènes pour mettre au jour de nouveaux concepts (mortalité, salubrité de l'air, longévité ...) identifiés et utilisés par l'administration sanitaire étatique. Cette nouvelle technologie suppose l'hôpital comme administrateur de la santé pour l'État à l'échelle d'un district.

61 Kreager 1988, 129, 134; 2015, S30.

62 Goody 1979.

63 Hess, Mendelsohn 2010. 
De fait, les techniques comptables et la mise sous forme de tables permettent de repérer des constantes sérielles. John Graunt (1620-1674) applique le premier des pratiques marchandes à des sujets politiques, notamment l'épidémiologie et la puissance de la nation anglaise, grâce à des registres de données qu'il traite de façon arithmétique. Il met en place des tables de mortalité, à partir du recueil des décès et de leurs causes au niveau des paroisses londoniennes. ${ }^{64} \mathrm{Il}$ cherche à repérer le point de basculement de l'endémie à l'épidémie. Collègue et ami de Sydenham et Locke, William Petty (1623-1687) prolonge ces travaux pionniers ${ }^{65}$ et conteste de façon systématique le modèle épistémologique de la géométrie, au profit d'un modèle arithmétique dès les années $1648-1651 .{ }^{66}$ La connaissance médicale ne dépend pas tant d'idées et de démonstrations, que des observations quantifiées en termes de «nombre, poids et mesure» en vue d'améliorer les conditions d'existence. ${ }^{67}$ Petty joue un rôle important pour développer les procédures d'analyse mathématique des recensions ${ }^{68}$ et les appliquer non seulement en clinique, mais aussi en économie ${ }^{69}$ et en démographie. ${ }^{70}$ Reprenant à son compte l'intuition de Bacon, qui assigne à la médecine l'objectif de promouvoir la croissance de la population et l'amélioration des conditions d'existence de façon rationnelle, ${ }^{71}$ il découvre des relations arithmétiques entre le nombre de morts, de naissances, les classes d'âges dans le Discourse Made before the Royal Society the 26. Of November 1674 concerning the use of duplicate proportion (London, 1674).

Contestant la médecine classique inefficace, ${ }^{72}$ Petty propose d'établir une «anatomie politique», c'est-à-dire une médecine à l'échelle de la population, fondée non plus sur un modèle géométrique idéal, mais sur une arithmétique manipulant des données empiriques mises en séries. ${ }^{73}$ Graunt proposait des techniques comptables, Petty généralise et affine les outils mathé-

64 Rohrbasser 1999.

65 Hull 1899, 459, n.1, Kreager 1988, Rohrbasser 2009. La question de savoir qui était l'auteur des Natural and political observations, mentioned in a following Index, and made upon the bills of mortalité a été disputée: Landsdowne et Greenwood (1928) ou Groenewegen (1967) les attribuent à Petty, mais Dupâquier (1984) et McCormick (2009,131-132) ont argumenté de façon convaincante qu'il s'agissait de Graunt. Sur les relations entre Petty et Graunt: Mc Cormick 2009, 131-135.

66 Mc Cormick 2009, p. 5, 303-305.

67 Petty [1690], Preface. 1899, vol. 1, 244.

68 Mc Cormick 2009, 53-56, 61-62.

69 Aspromourgos 2001, Mc Cormick 2009, 135-147.

70 Reungoat 2004.

71 McCormick 2009, 120, 129-134, 177-179, 187-189, 201-208, 216-230; Slack 2015, 120.

72 Mais il critique aussi la science cartésienne inutile (qu'il a étudiée à Utrecht et Leyde), dans une lettre à Henry More, 1648.

73 W. Petty forge le terme d'arithmétique politique dans les années 1672 (Slack 2004). 
matiques $:{ }^{74}$ surtout, il les déploie systématiquement dans un projet politique de colonisation de l'Irlande dans le cadre de la reconquête par Cromwell en 1672, en vue d'améliorer la situation économique du pays. ${ }^{75}$ L'Irlande constitue ainsi le premier terrain d'application de ce projet à la fois théorique et pratique, pour établir une nouvelle règle de calcul, non plus la norma du géomètre, mais le calcul des relations dupliquées susceptible de relier des données, telles que le nombre de femmes, leurs âges et le nombre d'enfants nés, ou encore, le nombre de morts par type de causalité, les conditions sanitaires et la production économique d'une région. ${ }^{76}$

Pour réaliser ce projet, il faut passer de l'homo quadratus au corps politique perfectionné, de l'anatomie classique à l'anatomie politique. Les concepts de symétrie, fabrique ou proportion n'ont plus alors un sens géométrique et architectural, mais arithmétique et politique:

Sir Francis Bacon, in his Advancement of Learning hath made a judicious parallel in many particulars, between the Body Natural and Body Politick, and between the Arts of preserving both in Health and Strength. And it is reasonable, that as Anatomy is the best foundation of one, so also of the other; and that to practice upon the Politick, without knowing the Symmetry, Fabrick, and Proportion of it, is as casual as the practice of Old women and Empyricks. (William Petty, The political anatomy of Ireland London, Robert Clavel, 1691. Je souligne.)

En déplaçant les critères classiques de la santé vers le corps politique (Body politick), Petty les transforme en leur conférant désormais une pertinence arithmétique..$^{77} \mathrm{La}$ rationalité change: elle ne se détermine plus en référence à un idéal, au même titre que les idées géométriques servent de règle pour guider l'architecte dans ses constructions, mais elle exprime les relations constantes entre des quantités mesurant l'occurrence d'un phénomène empirique: des lois, rules ou laws, que l'homme politique utilise pour prendre des décisions et imposer des lois civiles.

Cette conception renvoie non plus au corpus antique architectural, mais juridique qui reprend le système d'équivalences entre norma, règle, gnomon et loi: contemporain de Vitruve et cherchant comme lui à constituer la spécificité de la rationalité et la langue latines à partir de la traduction des concepts et termes grecs, Cicéron discute l'étymologie de la «lex» traduction du grec «nomon» (nomine nomon). Dans le domaine de la justice, il repère la même ambiguïté que Vitruve entre, d'une part, une loi parfaite, rationnelle et naturelle, idée de la loi naturelle, qui est la règle du juste de l'injuste (juris atque iniuriae regula), et, d'autre part, les lois civiles choisies par une

74 Mc Cormick 2009, 5, 303-305.

75 Mc Cormick 2009, 135-147, 168-196, Slack 2015, 120.

76 Kreager 1988, 129.

77 Mc Cormick 2009, 53-56, 61-62. 
société, comme les Douze Tables à Rome. ${ }^{78}$ Le terme français de règle ou anglais de rule conserve cette double étymologie ambiguë, qui renvoie simultanément au droit (et le pouvoir de régir du roi) et à l'instrument mathématique..$^{79}$ La nouvelle rationalité scientifique permet alors d'ajuster les lois de la nature et les règles politiques pour guider l'action humaine de façon juste et certaine, c'est-à-dire efficace. Les lois politiques correspondront ainsi aux lois de la nature, de façon empirique, puisqu'elles en émanent et s'attachent à orienter leurs logiques vers un intérêt précis. ${ }^{80}$ Ainsi par exemple, il ne faut pas contrecarrer la logique de l'usure, qui répond aux attentes naturelles et logiques des prêteurs, mais l'encadrer juridiquement. De façon générale, il convient de respecter le sens commun et de ne considérer que les causes qui ont visiblement des fondations naturelles. ${ }^{81}$ L'anatomie politique retrouve alors les concepts de symmetria, fabrique et proportion, mutatis mutandis, à partir de règles arithmétiques.

Ce déplacement induit une nouvelle idée de la règle, non plus la norma du géomètre mais la décision politique fondée sur une analyse quantitative des paramètres à l'échelle d'une population. ${ }^{82}$ La santé publique devient ainsi une affaire d'État, faisant converger ainsi les enjeux scientifiques et politiques: «the state - the agent of policy - became a scientific agent as well, promoting growth through mechanical improvements and institutional innovations». ${ }^{83}$ Savoir signifie alors assembler des données hétérogènes «discerning an inherent order beneath the manifold flow of events», ${ }^{84}$ comparer une situation à une autre, des scénarii entre eux et prendre une décision en termes d'optimisation, l'efficacité devenant ainsi le nouveau critère de la justesse et de la justice d'une action politique, qui repose sur un calcul arithmétique des relations régulières. ${ }^{85}$

Selon cette nouvelle épistémologie, se met en place un réseau, qui opère à trois niveaux: des enquêtes sanitaires systématiques à l'échelle de la population, le traitement des données d'un point de vue conceptuel et mathématique, la collaboration entre des médecins, des philosophes et des hommes politiques. ${ }^{86}$ Grâce à l'arithmétique politique appliquée à la santé, la croissance et le bien-être de la population, la médecine devient une affaire

78 Cicéron, De legibus I, 6, 19; Ernout-Meillet 2001; Jucquois 1998, 81-82.

79 Cassin 2004, § Règle, 1069.

80 Daston et Stolleis 2008.

81 «to use only arguments of sense, and to consider only such causes, as have visible foundations in nature» Political arithmetick, Preface, 1690.

82 Mc Cormick 2009, 9, 119-167, 298-300, Reungoat 2004, 190-192.

83 Mc Cormick 2009, 146.

84 Kreager 1988, 137.

85 Kreager 1988, 138-140; 2015, S32; Slack 2004, 43.

86 Hacking 1975, Shapiro 1983. 
d'Etat. ${ }^{87}$ Ce genre d'enquête s'étend progressivement à travers l'Europe..$^{88}$ Avec ces travaux, la probabilité sort du domaine de l'opinion pour entrer dans le champ de la science fondé sur une méthodologie. ${ }^{89}$ Cette nouvelle épistémologie de la connaissance médicale implique quatre effets majeurs: elle modifie la conception de la santé; elle recompose les relations entre Etat, universités, académies et hôpitaux; elle confère un nouveau statut public aux données sanitaires dont s'empare l'Etat, et elle institue un débat public sur les concepts et méthodes requis pour mener à bien ce projet, ainsi que des comparaisons stratégiques entre les nations ou entre différentes catégories de la population (selon l'âge, le sexe, la richesse, la profession, les conditions de vie, etc.). La santé devient une res publica. La collaboration nouvelle entre médecins et hommes politiques conduit à des régulations économiques et légales..$^{90}$

La science médicale s'ancre désormais dans des expériences ordinaires mises en séries, des inductions, des probabilités, plutôt qu'en des idéaux et des certitudes. La quantification permet de décider où et quand la maladie passe de l'endémie à l'épidémie, et quelles mesures doivent prendre l'hôpital, l'Etat ou la paroisse pour prévenir ou endiguer la contagion. La quantification et la typologie des maladies permettent d'évaluer et améliorer la qualité de l'air, des hôpitaux, des villes. Il y a bien démonstration, mais selon une méthode arithmétique plutôt que géométrique, fondée sur des observations pratiques et validées a posteriori par leur efficacité. Enfin, les critères d'évaluation du savoir relèvent désormais de la politique publique et de la justice, selon leur utilité. ${ }^{91}$

Le changement induit par l'outil d'arithmétique politique suscite une transformation du concept de santé, qui s'éloigne de la règle géométrique ou architecturale et s'approche de la régularité au sens de fréquence tout en se liant au champ des lois mécaniques de la nature et des lois civiles de la politique. Il se déplace de l'individu vers la population, de l'idée vers les faits. La santé doit désormais décrire un état réel de la population censé ne mobiliser aucune métaphysique en appliquant une méthode empirique à des groupes humains inscrits dans des contextes sociaux (une ville, une classe d'âge, un sexe, une profession, etc.). Elle requiert de nouveaux termes et concepts, comme la longévité, l'espérance de vie, la fertilité, la moyenne d'âge, la mortalité, fondés sur des observations quantitatives de la popula-

87 Dupâquier 1985, Desrosières 1993, Brian 1994, Slack 2015, 100-133.

88 Faure 1997, Porter 1994, 1999, 45-76.

89 Kreager 1988, p. 129. Parmentier 1995.

90 Buck 1977, Mc Cormick 2009, 119-163.

91 Kreager 1988, 138-140; 2015, S32; Slack 2004, 43 Slack 2004, 43. 
tion. ${ }^{92}$ Elle doit aussi servir d'indicateur et de critère de décision pour des politiques publiques menées par l'Etat en termes de salubrité et de cordon sanitaire. Une politique de santé publique (political medicine ${ }^{93}$ ) se met en place à travers des institutions et des médecins salariés organisés en réseau, en promouvant l'augmentation de la natalité, de la longévité, de la salubrité grâce à des améliorations techniques et des innovations institutionnelles. ${ }^{94}$ Connaître ne signifie plus découvrir des causes fondamentales ou une essence du corps humain, mais créer de l'ordre en agrégeant et en distinguant des maladies semblables, analogues ou distinctes. La connaissance est orientée par l'action, et, plus précisément, l'art de gouverner.

La constitution historique de la santé publique illustre ainsi l'alternative entre une conception classique de la médecine visant la santé de l'individu comme un idéal et une approche pragmatique qui fait de la médecine une affaire politique et économique à l'échelle de la population. Leibniz ou Petty en ont tout à fait conscience. Pour le philosophe de Hanovre, l'arithmétique politique s'avère nécessaire pour faire de la médecine une science capable de traiter la contingence en constituant son objet à partir de la distribution de symptômes dans des groupes de population donnés. ${ }^{95}$ Elle comble un point aveugle de la médecine et permet de légitimer des actions et des objectifs en discernant un ordre intrinsèque des choses sous le flux complexe des événements. ${ }^{96}$ De façon assez paradoxale pour la science de l'époque, ces auteurs affirment qu'en médecine évaluer la réalité requiert la probabilité et doit se distinguer des autres savoirs fondés sur la démonstration géométrique, c'est-à-dire la certitude. ${ }^{97}$

La reconnaissance de la légitimité de l'arithmétique politique en médecine en France a pris plus de temps: au-delà des problèmes techniques dus à la difficulté de développer les outils mathématiques correspondants, les concepts de santé publique et les réseaux professionnels nécessaires pour recueillir et traiter les données, le défi s'avère d'abord conceptuel et épistémologique..$^{98}$ Elle déstabilise aussi l'institution médicale universitaire héritière du MoyenÂge et le monopole de l'université. Un nouvel ordre d'évaluation scientifique se met en place, attentif aux implications sociales et politiques du savoir. ${ }^{99} \mathrm{La}$

92 Leibniz 1680, Essay de quelques raisonnemens nouveaux; Quaestiones calculi politici circa Hominum cognotae, Parmentier 1995.

93 Petty, Letter to the Earl of Anglesey, December 1672, BL MS Add. 72858, f. 73, cité par T. Mc Cormick.

94 Mc Cormick 2009, 146.

95 Leibniz 2004, 91; Leibniz 1680, A IV III, 457.

96 Kreager 1988, 137.

97 Leibniz 2001, § XXII; Bouveresse 2010, 254; Rey 2000, 299.

98 Hacking 1975; Shapiro 1983; Banta 1987, Rey 2000.

99 Ben Chaim 2004, 5, 15, 101-141. 
médecine devient un sujet public discuté à des niveaux thérapeutique, épistémologique, politique, religieux et économique. L'Etat doit garantir la distribution des médecins sur le territoire, contrôler les épidémies; il assume davantage de responsabilités dans la prise en charge des pauvres ou des personnes âgées non seulement par charité, mais aussi par intérêt bien compris. ${ }^{100}$

\section{Conclusion}

L'épistémologie historique met en lumière le feuilletage conceptuel, ${ }^{101}$ qui s'insinue dans le concept de santé à travers l'émergence d'une nouvelle épistémologie liée à l'arithmétique politique et l'enrichissement ontologique de l'anatomie à travers la constitution d'un nouvel objet, le corps politique - plus tard, la population - que traite la médecine politique. Cette ontologie et cette épistémologie médicales répondent à un nouveau style de raisonnement médical, qui se stabilisera à travers les statistiques. ${ }^{102}$ La détermination de la santé des corps singuliers passe de la comparaison avec la règle, le canon ou l'idéal qui sert de référence absolue pour tous les cas singuliers, aux faits ordinaires et fréquents pour un groupe déterminé. La coexistence de ces deux épistémologies et ontologies révèle l'épaisseur des concepts de santé et la coexistence de plusieurs styles de rationalité scientifique pratiqués par les médecins modernes. ${ }^{103}$ Chaque style - géométrique ou arithmétique - embarque des objets scientifiques distincts (la fabrique du corps humain ou la population) relevant de plusieurs ontologies, avec des définitions de règle ou de loi distinctes, et s'inscrit dans deux lignées historiques - l'une ancrée dans la continuité antique conservée par l'université pour l'anatomie classique, l'autre ouvrant une modernité hospitalière et étatique pour l'anatomie politique - qui se nouent ensemble dans l'histoire longue de la médecine moderne occidentale. ${ }^{104} \mathrm{Le}$ pluralisme scientifique désigne la cohabitation de plusieurs formes de rationalités scientifiques. Ce pluralisme ne constitue pas un accident ou un aléa de

100 Carvallo 2010, Lawrence 1996, Petty 1899, vol.1, 241-245, 300-302.

101 Ruphy 2011. Stéphanie Ruphy traite de questions contemporaines; Ian Hacking s'intéresse à l'histoire longue; de fait, ces catégories valent pour la science moderne plus largement.

102 Hacking 1992, Daston 2008, 1115.

103 Les deux styles présentés ici correspondent aux styles 1 et 5 déterminés par Crombie et repris par Hacking: 1) l'établissement de postulats sur le modèle des mathématiques grecques; 5) l'analyse statistique des régularités au sein d'une population et le calcul des probabilités. Crombie 1994; Hacking 1992.

104 Hacking établit 4 critères caractérisant un style scientifique: l'introduction de nouveaux objets et types de propositions ou de lois; la capacité d'un style à établir ses propres critères de vérification; la stabilisation historique d'un style; son ancrage dans un contexte culturel. Hacking 1992. 
l'histoire que l'on pourrait corriger, mais fait partie de la dynamique scientifique, dans la mesure où elle suscite de nouveaux candidats à la vérité et la fausseté en faisant émerger de nouveaux statuts de la vérité, de nouveaux objets, de nouvelles règles. Certes, à chaque époque domine un paradigme privilégié, mais il n'étouffe pas la réalité foisonnante de la science, qui ne se réduit pas à une simple juxtaposition, mais introduit véritablement un feuilletage conceptuel et pratique. Dans une approche maximaliste, qui tient compte de l'épaisseur des concepts constitutifs de la science, oui, il y a un enrichissement ontologique et épistémologique de la médecine dans les années 16501680 , car s'opère un changement en profondeur du rapport à la norme qui permet de passer des corps singuliers au concept général de santé. Une enquête terminologique et conceptuelle sur la règle le prouve en mettant au jour le sens architectural - esthétique, plus largement - de la règle-norma, par différence avec l'émergence d'un sens politique et populationnel.

\section{Bibliographie}

Ackerknecht, Erwin H. A short history of medicine (Baltimore et Londres [1959] 1968)

Aersten, Jan Arsten, Medieval philosophy and the Transcendantals: the case of Thomas Aquinas (Leyde 1996)

Alembert, d', Jean, Diderot, Denis, Encyclopédie, ARTFL Encyclopédie Project, éd. Robert Morrissey

Amati, Frank, Tony Aspromourgos, Petty contra Hobbes: A previously Untranslated Manuscript, Journal of the History of Ideas 46 (1985) 127-132

Anstey, Peter R., «Experimental versus Speculative Natural philosophy», dans: P.R. Anstey and J.A. Schuster(éds), The science of Nature in the Seventeenth Century (Springer, 2005), 215-242

Anstey, Peter R., John Locke and Natural Philosophy (Oxford 2011a)

Anstey, Peter R., «The Creation of the English Hippocrates», Medical History 55 (2011b) 457-478

Anstey, Peter R., Lawrence M. Principe, John Locke and the case of Anthony Cooper, Early Science and Medicine 16 (2011) 379-503

Aspromourgos, Tony, The mind of the Oeconomist: An overview of Petty Papers Archive, History of Economical Ideas 9 (2001) 79-83

Aviler, Augustin C., D', Explication des termes d'architecture qui concernent l'architecture, les mathématiques [...] Ensemble les Etimologies et les Noms latins des termes (Paris 1691)

Aucante, Vincent, La philosophie médicale de Descartes (Paris 2006)

Azouvi, François, Entre Descartes et Leibniz: l'animisme dans les Essais de Physique de Claude Perrault, Recherches sur le XVIIe siècle 5 (1982) 9-19

Ballester, Luis Garcia, Galen and Galenism: theory and medical practice from antiquity to the European Renaissance (Burlington 2002) 
Banta, James E., «Sir William Petty: modern epidemiologist (1623-1687)», Journal of Community Health 12 (1987) 185-198

Baratay, Eric, Claude Perrault (1613-1688), observateur révolutionnaire des animaux, XVIIe siècle. 255 (2012) 309-320

Ben-Chaim, Michael, Experimental Philosophy and the Birth of empirical Science. Boyle, Locke and Newton (Aldershot 2004)

Bertin, Dominique, Ian Gardet Bourbonnois, Epitome, ou Extrait abrege des dix livres d'architecture de Marc Vitruve Pollion (Toulouse 1556)

Bird, Alexander, «Kuhn on Reference and Essence», Philosophia Scientiae 8 (2004) 39-71

Boorse, Christopher, On the distinction between disease and illness, Philosophy and Public Affairs 5 (1975) 49-68

Brenner, Anastasios, «Quelle épistémologie historique? Kuhn, Feyerabend, Hacking et l'école bachelardienne», Revue de Métaphysique et de morale 49 (2006) 113-125

Brian, Eric, La mesure de l'Etat (Paris 1994)

Bouveresse, Jacques, Dans le labyrinthe: nécessité, contingence et liberté chez Leibniz: Cours 2009 et 2010 (Paris 2010) (généré le 20 mai 2016) <http://books. openedition.org/cdf/1785>. ISBN: 9782722601611

Buck, Peter, Seventeenth Century Political Arithmetic; Civil Strife and Vital Statistics, Isis 68 (1977) 67-84

Cantor, David (éd.), Reinventing Hippocrates (Aldershot 2002)

Canguilhem, George, Le normal et le pathologique (Paris 1966)

Carey David, Compiling nature's history: Travellers and travel narratives in the early Royal Society, Annals of science 54 (1997) 269-292

Carvallo, Sarah, La circulation sanguine comme pierre de touche. Harvey, Riolan, Descartes, Lato Sensu (2016) 85-92: http://dx.doi.org/10.20416/lsrsps.v3i1.243

Carvallo, Sarah, L'homme parfait - L'anthropologie médicale de Harvey, Riolan et Perrault (1628-1688) (Paris 2017)

Carvallo, Sarah, L'anthropologie platonisante de Riolan, dans: Mai Lequan, Anne Merker, Jean-Michel Counet (éds), La pensée en devenir. La réception du platonisme et du néoplatonisme dans l'histoire de la philosophie (Louvain, à paraître)

Carvallo, Sarah, The empirical Turn of Medicine in England 1660-1690, Archives internationales d'histoire des sciences 67 (2017) 75-112

Carvallo, Sarah, Ageing in the Seventeenth and Eighteenth Centuries, Science in Context 23 (2010) 267-288

Cassin, Barbara (éd.), Vocabulaire européen des philosophies. Dictionnaire des intraduisibles (Paris 2004)

Caye, Pierre, Science et efficience: la métaphysique d'Aristote à l'épreuve du De architectura de Vitruve, Corpus 39 (2001) 133-154

Cicéron, De natura deorum, éd. par Martin van Bruwaene (Bruxelles [44] 1970)

Cicéron, La nature des dieux, éd. par Clara Auvray-Assayas (Paris 2004)

Cicéron, L'Orateur, éd. par Albert Yon (Paris 1964)

Cook, Harold J., The Decline of the Old Medical Regime in Stuart London (Ithaca-NY 1986)

Cook, Harold. J., The New Philosophy and Medicine in seventeenth-Century England, dans: David Lindberg, Robert Westman (éds), Reappraisal of the Scientific Revolution (Cambridge 1990) 397-436 
Cook, Harold J., Good Advice and Little Medicine: The Professional Authority of Early Modern English Physicians, Journal of British Studies 33 (1994) 1-31

Cook, Harold J., Medicine, dans: Katherine Park et Lorraine Daston (éds), The Cambridge History of Science. Early Modern Science (Cambridge 2008) vol. 3, 407-435

Cook, Harold J. Victories for Empiricism, Failures for theory, dans: Charles T. Wolfe, Ofer Gal (éds), The Body as Object and Instrument of Knowledge. Embodied empiricism in early modern science (Dordrecht 2010) 9-32

Crane, Judith K., Locke's theory of classification, British Journal for the History of Philosophy 11 (2003) 249-259

Crignon, Claire, The debate about methodus medendi during the Second Half of the Seventeenth Century in England: Modern Philosophical Readings of Classical Medical Empiricism in Bacon, Needham, Willis and Boyle, Early Science and Medicine 18 (2013) 13-33

Crignon, Claire, Nunzio Alloca, Carsten Zelle, Medical Empiricism and Philosophy of Human Nature in the $17^{\text {th }}$ and $18^{\text {th }}$ Century, Early Science and Medicine 18 (2013) 1-12

Crignon, Claire, Révolution anatomique et révolution cosmologique: quelques réflexions à partir de la lecture de «l'homme de Vésale dans le monde de Copernic», Revue de Métaphysique et de morale 82 (2014) 167-195

Crombie, Alistair, Styles of scientific thinking in the European tradition (Londres 1994)

Cunningham, Andrew, Thomas Sydenham: Epidemics, Experiment and the «good old cause», dans: Roger French et A. Wear (éds), The medical Revolution of the Seventeenth Century (Cambridge 1989) 164-190

Cunningham, Andrew, The transformation of Hippocrates in Seventeenth-century Britain, dans: David Cantor (éd.), Reinventing Hippocrates (Aldershot 2002) 91115

Cunningham, Andrew, The anatomical Renaissance. The resurrection of the anatomical projects of the anciens (Aldershot [1997] 2003)

Daston Lorraine, Probability and evidence, Cambridge History (Cambridge 2008)

Daston Lorraine, Michael Stolleis, Natural Law and Laws of Nature in early modern Europe. Jurisprudence, Theology, moral and natural philosophy (Londres, 2008)

Dear, Peter, The meaning of experience, dans: Katharine Park et Lorraine Daston (éds), The Cambridge History of Science. Early Modern Science (Cambridge[2006] 2008) vol.3, 106-131

Des Chene, Denys, Physiologia: Natural Philosophy in Late Aristotelician and Cartesian Thought (Ithaca 1996)

Des Chene, Denys, Mechanisms of Life in the Seventeenth Century: Borelli, Perrault, Régis, History and Philosophy of Biological and Biomedical Sciences 36 (2005) 245-260

Desrosières Alain, La politique des grands nombres. Histoire de la raison statistique (Paris 1993)

Dewhurst, Kenneth, «Locke and Sydenham on the teaching of Anatom», Medical History. 2 (1958) 1-12

Diderot, Denis, D'Alembert, J. Encyclopédie ou Dictionnaire raisonné des Sciences, des arts et des métiers (Paris 1751-1772), Ou http://portail.atilf.fr/encyclopedie/Dictionnaire universel françois et latin, ou Dictionnaire de Trévoux (Paris [1721] 1771) 
Distelzweig, Peter, «Mechanics» and Mechanism in William Harvey's Anatomy: Varieties and Limits, dans: Peter Distelzweig, Benjamin Goldberg, Enan R. Ragland (éds.), Early Modern Medicine and Natural Philosophy (Dordrecht 2016) $117-140$

Duchesneau, François, Les modèles du vivant de Descartes à Leibniz (Paris 1998)

Dupâquier, Jacques, William Petty et l'invention de la table de mortalité, Population 39 (1984) 1069-1073

Dupâquier, Jacques, Michel Dupâquier, Histoire de la démographie (Paris 1985)

Engelhardt, Tristram H. Jr., "Concepts of Health and Disease», dans id. et Stuart F. Spicker (éds), Evaluation and Explanation in the Biomedical Sciences (Dordrecht 1975) 125-141

Ernout, Alfred Meillet, A., Dictionnaire étymologique de la langue latine. Histoire des mots (Paris [1951] 2001)

Faure, Olivier, Les stratégies sanitaires, dans: Mirko D. Grmek (éd.), Histoire de la pensée médicale (Paris 1997) t. 2, 279-296

Foucault, Michel, Naissance de la Clinique. Une archéologie du regard médical (Paris 1963)

Foucault, Michel, Sécurité, territoire, population. Cours au collège de France 197778, éd. par Michel Senellart (Paris 2004)

French, Roger, William Harvey's natural Philosophy (Cambridge 1994)

French, Roger, Dissection and Vivisection in the European Renaissance (Aldershot 1999)

Fumaroli, Marc, «L'apologétique de la langue française classique», Rhetorica 2 (1984) 139-161

Furetière, Antoine, Dictionnaire universel contenant generalement tous les mots françois, tant vieux que modernes, et les termes de toutes les sciences et des arts (La Haye-Rotterdam 1690)

Galien, Opera Omnia, éd. par C.arl Gottlob Kühn (Leipzig 1821-1833)

Galien, Exhortation à l'étude de la médecine. Art médical, éd. par Véronique Boudon (Paris 2000)

Garcia-Ballester, Luis, Galen and Galenism: theory and medical practice from antiquity to the European Renaissance (Burlington 2002)

Geertz, Clifford, The interpretation of culture (New York 1973)

Goody, Jack, La raison graphique. La domestication de la pensée sauvage (Paris 1979)

Graunt, John, Natural and Political Observations Mentioned in a following Index, and made upon the Bills of mortality (London, 1662), dans: Charles Henry Hull (éd.), The Economic Writings of Sir William Petty (Cambridge 1899)

Grenier, Jean-Yves, André Orléan, «Michel Foucault, l'économie politique et le libéralisme», Annales. Histoire, Sciences sociales (2007) 1155-1182

Groenewegen, Peter D., «Authorship of the Natural and Political Observations Upon the Bills of Mortality» Journal of the History of Ideas 28 (1967) 601-602

Gros, Pierre, Vitruve et la tradition des traités d'architecture. Fabrica et ratiocinatio (Ecole française de Rome 2006)

Gros, Pierre, «La basilique de forum selon Vitruve: la norme et l'expérimentation», dans: Vitruve et la tradition des traités d'architecture. Fabrica et ratiocinatio (Rome 2006) 217-237 
Guerrini, Anita, The king's animals and the king's books: the illustrations for the Paris Academy's Histoire des animaux, Annals of Science 67 (2010) 383-404

Hacking, Ian, The emergence of Probability (London-New York 1975)

Hacking, Ian, Statistical language, statistical truth and statistical reason: the self-authentification of a style of scientific reasoning, dans: Ernan McMullin (éd.), The social dimension of science (Notre Dame Ind. 1992) 130-157

Harcourt, Glenn, «Andreas Vesalius and the Anatomy of Antique Sculpture», Representations 17 (1987) 28-61

Hermann, Wolfgang, La théorie de Claude Perrault (Bruxelles 1980)

Hess, Volker, J. Andrew Mendelsohn, Case and series: medical knowledge and paper technology, 1600-1900, Science History, 48 (2010) 287-314

Hess, Volker, J. Andrew Mendelsohn, «Fallgeschichte Historia, Klassifikation. François Boissier de Sauvages bei der Schreibarbeit», NTM. Zeitschrift für Geschichte der Wissenschaften, Technik und Medizin 21 (2013), 61-92

Hirai, Hiro, Alter Galenus: Jean Fernel et son interprétation platonico-chrétienne de Galien, Science and Medicine 10 (2005) 1-35

Hirai, Hiro, Lecture néoplatonicienne d'Hippocrate chez Fernel, Cardan et Gemma, dans: Jacqueline Vons (éd.), Pratique et pensée médicales à la Renaissance (Paris 2009) 91-110

Hirai, Hiro, Medical Humanism and Natural Philosophy: Renaissance Debates on Matter, Life and the Soul (Leyde 2011)

Hull, Charles H., The economic writings of Sir William Petty together with the Observations upon the Bills of Mortality more probably by Captain John Graunt (Cambridge 1899)

Jacquart, Danièle, La Médecine médiévale dans le cadre parisien XIVe-XVe siècles (Paris 1998)

Jucquois, Guy, Echanges et codifications des règles, dans: Yves Duhoux (éd.), Hommage à Albert Maniet (Louvain 1998) 69-97

Jutel, Annemarie, Classification, Disease, and Diagnosis, Perspectives in Biology and Medicine 54 (2011) 189-205

Kreager, Philip, New light on Graunt, Population studies 42 (1988) 129-140

Kreager, Philip, Population theory - A long view, Population Studies 69: suppl. (2015) S29-S37, DOI: 10.1080/00324728.2014.981095

Kuhn, Thomas, Commensurability, communicability, comparability, dans: id., The Road since Structure (Chicago 2000) 33-57

Kuhn, Thomas, Dubbing and Redubbing: The Vulnerability of rigid Designation, dans: C. Wade Savage, James Conant, John Haugeland (éds), Minnesota Studies in the Philosophy of Science (University of Minnesota Press 1990) 58-89

Laneyrie-Dagen, Nadèje, L'invention du corps. La représentation de l'homme $d u$ Moyen Âge à la fin du XIXe siècle (Paris 2006)

Lawrence, Susan C., Charitable Knowledge: Hospital pupils and practitioners in eighteenth century in London (Cambridge 1996)

Leibniz, Gottfried W., «Essay de quelques raisonnements nouveaux 1680», dans: Sämtliche Schriften und Briefe (Berlin-Darmstadt 1923)

Leibniz. Gottfried W., Discours de métaphysique et autres textes 1663-1689, éd. par Christiane Frémont (Paris 2001) 
Leibniz, Gottfried W., Controverse sur la Vie, l'Organisme et le Mixte, éd. par Sarah Carvallo (Paris 2004)

Lemerle, Frédérique, «Vitruve, Vignole, Palladio au dix-septième siècle: traductions, abrégés et augmentations», dans: Jean-Philippe Garric, Frédérique Lemerle, Yves Pauwels (éds), Architecture et théorie. L'héritage de la Renaissance (Paris 2009)

Locke, John, The Clarendon edition of the Works of John Locke, 20 vols (Oxford 1976-2012)

Mandressi, Rafael, Le regard de l'anatomiste (Paris 2003)

Mandressi, Rafael, Métamorphoses du commentaire. Projets éditoriaux et formation du savoir anatomique au XVIe siècle, Gesnerus 62 (2005) 165-185

Mandressi, Rafael, Médecine et discours sur l'homme dans la première modernité, Revue de synthèse 134 (2013) 511-536

Mc Cormick, Ted, William Petty and the Ambitions of Political Arithmetic (Oxford 2009)

MacHugh, Tim, Hospitals Politics in Seventeenth Century France. The Crown, Urban Elite and the Poor (Aldershot 2007)

Mani, Nikolas, Jean Riolan II (1580-1657) and Medical Research, Bulletin of the History of Medicine 42 (1968) 121-144

Martensen, Robert L., «Hippocrates and the Politics of medical knowledge in early modern England», dans: David Cantor (éd.), Reinventing Hippocrates (Aldershot 2002) 116-135

Mazauric, Simone, Fontenelle et l'invention de l'histoire des sciences à l'aube des Lumières (Paris 2007)

Meli, Domenico B., Machines of the Body in the Seventeenth Century, dans: in Peter Distelzweig, Benjamin Goldberg, Evan R. Ragland (éds), Early Modern Medicine and Natural Philosophy (Dordrecht 2016) 91-116

Meynell, Guy G., John Locke and the Preface to Thomas Sydenham's Observationes Medicae, Medical History 50 (2006) 93-110

Nutton, Vivian, God, Galen and the Depaganization of Ancient Medicine, dans: Peter Biller et Joseph Ziegler (éds), Religion and Medicine in the Middle Ages (York 2001) 17-32

Pagel, Walter, William Harvey's Biological Ideas. Selected Aspects and Historical Backgrounds (Bâle 1967)

Panofsky, Erwin, Idea. Contribution à l'histoire du concept de l'ancienne théorie de l'art (Paris [1924] 1983)

Panofsky, Erwin, L'évolution d'un schème structural. L'histoire de la théorie des proportions humaines conçue comme un miroir de l'histoire des styles, dans: id. (éd.), L'œuvre d'art et ses significations. Essais sur les arts visuels, trad. par Bernard Teyssèdre (Paris 1969)

Papineau, David, Naturalism, dans: Edward N. Zalta (éd.), The Stanford Encyclopaedia of Philosophy (Stanford 2009) http://plato.stanford.edu/entries/naturalism

Parmentier, Marc, L'estime des apparences (Paris 1995)

Perrault, Claude, Les dix livres d'architecture de Vitruve corrigez et traduits nouvellement en françois, avec des notes et des figures (Paris 1673)

Perrault, Claude, Essais de Physique ou Recueil de plusieurs traitez touchant les choses naturelles. 4 vols (Paris 1680)

Perrault, Claude, Euvres diverses de Physique et de Méchanique (Leyde 1721) 
Petty, William, The Economic Writings of Sir William Petty, éd. par Charles Henry Hull, 2 vols (Cambridge 1899)

Petty, William, Political Arithmetick, Or a Discourse Concerning the extent and value of lands, people [...] (Londres 1690)

Petzet, Michael, Claude Perrault als Architekt des Pariser Observatoriums, Zeitschrift für Kunstgeschichte 30 (1967) 1-54

Picon, Antoine, Claude Perrault, 1613-1688, ou la curiosité d'un classique (Paris 1989)

Pigeaud, Jackie, Homo quadratus. Variations sur la beauté et la santé dans la médecine antique, Gesnerus 42 (1985) 337-352

Pigeaud, Jackie, Formes et normes dans le De Fabrica de Vésale, dans: Jean Céard, Marie-Madeleine Fontaine, Jean-Claude Margolin (éds), Le corps à la Renaissance (Paris 1990) 399-421

Pomata, Gianna, «Sharing Cases: The Observationes», Early Modern Medicine, 15 (2010) 193-236

Pomata, Gianna, Nancy Siraisi, Historia: Empiricism and Erudition in Early Modern Europe (Cambridge 2005)

Pommier, Edouard, Théories du portrait, De la Renaissance aux Lumières (Paris 1999)

Porter, Dorothy, Health, Civilization and the State. A history of public health from Ancient to Modern Times (Londres 1999)

Putnam, Hillary, The Collapse of the Fact/Value Dichotomy and Other Essays (Cambridge 2002)

Quine, Willard van O., Word and Object (Massachussets [1960] 2013)

Quine, Willard van O., Three Indeterminacies, dans: id. (éd.), Confessions of a confirmed Extensionalist: And other Essays (Massachussets 2008) 368-386

Reguig, Delphine (éd.), Dossier: Les frères Perrault, XVIIe siècle264 (2014)

Reungoat, Sabine, William Petty: Observateur des Îles Britanniques (Paris 2004)

Rey, Alain et al., Dictionnaire historique de la langue française (Paris [1992] 2006)

Rey, Roselyne, Naissance et développement du vitalisme en France de la deuxième moitié du 18e siècle (Oxford 2000)

Riolan, Jean, Anthropographia, et osteologia. Omnia recognita, triplo auctiora et emendatiora ex proprijs [...] (Paris 1626)

Riolan, Jean, Les æuvres anatomiques de Monsieur Jean Riolan, reveues [...] par M. Pierre Constant (Paris 1628-29)

Riolan, Jean, Opuscula anatomica nova, quae nunc in lucem prodeunt. Instauratio magna Physicae et Medicinae per novam doctrinam de motu circulatorio sanguinis in corde (Londres 1649)

Riolan, Jean, Manuel anatomique et pathologique, ou Abrégé de toute l'Anatomie et des Usages que l'on peut en tirer pour la connoissance, \& pour la guerison des Maladies (Paris 1661)

Rohrbasser, Jean-Marc, «William Petty (1623-1687) et le calcul du doublement de la population», Population 54 (1999) 693-706.

Romanell, Patrick, John Locke and Medicine: A new key to Locke (Buffalo NY 1984)

Roux, Sophie, Quelles machines pour quels animaux? Jacques Rohault, Claude Perrault, Giovanni Alfonsi Borelli, dans: B. Roukhomovsky, A. Gaillard, J. Y. Goffi, 
S. Roux (éds), L'Automate, Modèle, métaphore, machine, merveille (Bordeaux 2012) 69-114

Ruby, Jane, The origins of scientific «Law», Journal of the History of Ideas 47 (1986) 341-359

Ruphy, Stéphanie, From Hacking's plurality of styles scientific reasoning to a foliated pluralism: a philosophically robust form of ontologico-methodological pluralism, Philosophy of science 78 (2011) 1212-1222

Ryle, Gilbert, Thinking and reflecting, The Thinking of thoughts: what is «Le penseur» doing?, dans: id., Collected Papers, vol. 2 (Londres 1971) 465-496

Salomon-Bayet, Claire, L'institution de la science et l'expérience du vivant. Méthode et experience à l'Académie Royale des Sciences, 1666-1793, (Paris [1978] 2008)

Schmitt, Charles B., Aristote et la Renaissance (Paris [1983] 1992)

Schmitt, Charles B. ,Aristotle among Physicians, dans: Andrew Wear, Roger K. French, Iain M. Lonie (éds), The medical Renaissance of the Sixteenth Century (Cambridge 1985) 1-15

Sgarbi, Marco, The Aristotelian Tradition and the Rise of British Empiricism: Logic and Epistemology in the British Isles (1570-1689) (Dordrecht 2013)

Shapin, Steven, Possessed by the Idols. (David Wootton, Bad Medicine: Doctors Doing Harm since Hippocrates Reviewing), London Review of Books 28 (2006) 3133

Shapiro, Barbara J., Probability and certainty in Seventeenth Century England. A study of relationship between natural science, religion, history, law, and literature (Princeton 1983)

Siraisi, Nancy G., Medicine and the Italian universities 1250-1600 (Leyde et Boston 2001)

Siraisi, Nancy G., Medicine, 1450-1620, and the History of Science, Isis 103 (2012) 491-514

Slack, Paul, Government and Information in Seventeenth century England, Past and Present 184 (2004) 33-68

Slack, Paul, The invention of improvement. Information and material progress in seventeenth century England (Oxford 2015)

Sydenham, Thomas, The Works of Thomas Sydenham, éd. par Robert G. Latham, 2 vol. (Londres 1848-1850)

Temkin, Owsei, Galenism: Rise and Decline of a Medical Philosophy (Ithaca 1973)

Temkin, Oswald, The double face of Janus (Baltimore 1977)

Thomson, R. Paul, «Theories and models in medicine», dans: Fred Gifford (éd.), Philosophy of Medicine (Handbook of the Philosophy of Science, vol. 16) (Amsterdam 2011) 115-136

Tobin, Richard, The Canon of Polykleitos, American Journal of Archaelogy 79 (1975) 307-321

Van der Lugt, Maiike, Neither ill nor healthy: the intermediate state between health and disease in medieval medicine, Quaderni Storici 136 (2011) 13-46

Vitruvius, Les dix livres d'architecture de Vitruve, corrigez et traduits nouvellement en François avec des Notes et des Figures par Claude Perrault (Paris [1673] 1684)

Vitruvius, De l'Architecture, éd. par Pierre Gros (Paris 2015)

Vons, Jacqueline, Le rôle des analogies dans la transmission d'un savoir scientifique. André Vésale (1514-1564) et la Fabrica (1543), dans: Alfredo Perifano, Frank La 
Brasca (éds), La transmission des savoirs au Moyen-Âge et à la Renaissance (Besançon 2005) 33-48

Wakefield, Jerome, The concept of mental disorder: on the boundary between biological facts and social values, American Psychologist 47 (1992) 373-388

Wakefield, James, Fait et valeur dans le concept de trouble mental: le trouble en tant que dysfunction préjudiciable, Philosophiques 33 (2006) 37-63

Waldow, Anik, Empiricism and its roots in the Ancient Medical Tradition, dans: Charles T. Wolfe, Ofer Gal (éds), The Body as Object and Instrument of Knowledge. Embodied empiricism in early modern science (Dordrecht 2010) 287-308

Walmsley, Jonathan, Morbus. Locke's early Essay on disease, Early Science and Medicine, 5/4, 2000, 366-393

Wear, Andrew, Knowledge and Practice in english medicine 1550-1680 (Cambridge 2000)

Wilson, Catherine, Visual surface and visual symbol: the microscope and the occult in early modern science, Journal of the History of ideas 49 (1988) 85-108

Wolfe, Charles T., Empiricist heresies in early modern medical thought, dans: id., Ofer Gal, (éds), The Body as Object and Instrument of Knowledge. Embodied empiricism in early modern science (Dordrecht 2010) 333-344

Wolfe, David E., «Sydenham and Locke on the limits of Anatomy», Bulletin of the History of Medicine 35 (1961) 193-220

Wright, John P., Perrault's criticism of the Cartesian theory of the soul, dans: Stephen Gaukroger, John Schuster, John Sutton (éd.), Descartes' Natural Philosophy (Londres 2000) 680-696 